Research Article

\title{
Fractional-Order Fast Terminal Sliding Mode Control for a Class of Dynamical Systems
}

\author{
Guoliang Zhao \\ Modern Manufacture Engineering Center, Heilongjiang University of Science and Technology, Harbin 150027, China \\ Correspondence should be addressed to Guoliang Zhao; ocnzhao@gmail.com
}

Received 29 June 2013; Accepted 13 November 2013

Academic Editor: Guanghui Sun

Copyright ( 2013 Guoliang Zhao. This is an open access article distributed under the Creative Commons Attribution License, which permits unrestricted use, distribution, and reproduction in any medium, provided the original work is properly cited.

\begin{abstract}
This paper introduces a novel fractional fast terminal sliding mode control strategy for a class of dynamical systems with uncertainty. In this strategy, a fractional-order sliding surface is proposed, the corresponding control law is derived based on Lyapunov stability theory to guarantee the sliding condition, and the finite time stability of the closeloop system is also ensured. Further, to achieve the equivalence between convergence rate and singularity avoidance, a fractional-order nonsingular fast terminal sliding mode controller is studied and the stability is presented. Finally, numerical simulation results are presented to illustrate the effectiveness of the proposed method.
\end{abstract}

\section{Introduction}

In the past thirty years or so, fractional calculus has been found in numerous diverse and widespread fields of science and engineering [1-4] since the first fractional-order (FO) controller was introduced by Oustaloup in 1988 [1]. Thereafter, many different FO control strategies are studied, for example, fractional-order PID controller [5], FO leadlag compensators [6], and FO optimal controllers [7, 8]. Fractional-order control has made great progress in recent years, many researchers have endeavored to study different nonlinear systems, and several control methods and stabilities are proposed. To name a few, asymptotic stability is extended to Mittag-Leffler stability for nonlinear fractional neutral singular systems, and the newly proposed stability implies asymptotic stability $[9,10]$. A sliding mode controller with a new fractional-order integral switching surface for an uncertain chaotic fractional order economic system is developed; the sliding mode motion exists on every point of the switching surface and any state outside the surface is driven to the surface in a finite time [11]. Stabilization and synchronization of a class of fractional-order chaotic systems with a novel fractional-order sliding mode approach are thoroughly studied by Aghababa [12]. For a class of Lipschitz nonlinear fractional-order systems, a nonfragile observer is employed to tackle chaos synchronization problems [13]. For the open loop oscillatory systems, a novel conformal mapping based fractional-order methodology is developed to tune the classical PID controllers. The conventional pole placement tuning via linear quadratic regulator (LQR) method is also extended in some extent [14]. The primary resonance phenomenon of Duffing oscillator with fractionalorder derivative is researched by Shen et al. [15].

Recently, researchers have been using $\mathrm{H}_{\infty}$ control in fractional-order systems. In particular, Shen and Lama [16] proposed a state feedback $\mathrm{H}_{\infty}$ controller for commensurate fractional-order systems with a prescribed $\mathrm{H}_{\infty}$ performance. In the work of [17], a positive reduced-order fractional system is constructed, and the associated error system is stable with a prescribed $\mathrm{H}_{\infty}$ performance for a stable positive fractional-order system. For the fractionalorder systems with time-delay, Wang and Gao designed a fractional-order proportional-derivative (PD) controller with $\mathrm{H}_{\infty}$ performance [18]. Most recently, a novel fractionalorder sliding mode controller for output tracking of a time-varying reference signal is designed and the proposed method is applied to a fractional-order gyroscope model [19]. Consensus problems of fractional-order systems with nonuniform input and communication delays over directed static networks are studied based on a frequency-domain 
approach and generalized Nyquist stability criterion; some results are obtained to ensure the consensus of the fractionalorder systems [20]. For the consensus problems of delayed fractional-order systems and multiagent systems, see [21, 22] and the references therein. Furthermore, intelligent systems can also be incorporated into the fractional-order controller design. By using the chaotic ant swarm system, fractionalorder PID controller with objective function composed of overshoot, steady-state error, raising time, and settling time is studied [23].

Terminal sliding-mode control (TSMC) is a well-known efficient control scheme in the field of variable structure control, which has been widely applied to linear and nonlinear systems [24-28]. TSMC uses nonlinear sliding surfaces and the nonlinear switching hyperplanes can improve the transient performance substantially. Besides, compared with the conventional SMC with linear sliding surface, TSMC offers some superior properties such as finite time convergence and higher control precision. Dadras firstly introduces the fractional-order TSMC (FO-TSMC) to the integer-order nonlinear systems. However, there remain some unsolved problems in the paper [29], such as the chattering problem of control effort and the strict proof of convergence time to a given vicinity around the origin on the sliding surface.

In this paper, a new fractional-order fast terminal sliding surface is proposed to ensure fast convergence, a reaching law is developed to eliminate the chattering and theoretical proof of the finite time convergence is given. Moreover, a fractional-order nonsingular fast terminal sliding mode controller is proposed to achieve the fast convergence and eliminate chattering; integer-order plants with uncertainties and unmodeled dynamics are simulated to verify the newly proposed controller.

The rest of this paper is organized as follows. In Section 2, some preliminaries and definitions of fractional-order calculus are introduced. In Section 3, the system model under investigation and the problem formulation are presented. In Section 4, the FO-TSMC is briefly reviewed; a fractionalorder fast terminal sliding mode surface design and finite time stability analysis are done in Section 5. In Section 6, a fractional-order nonsingular fast terminal sliding mode surface design and stability analysis are researched. In Section 7, the effectiveness of the proposed control schemes are illustrated by numerical examples and the results are compared with the FO-TSMC. Finally, some concluding remarks are included in Section 8.

\section{Basic Definition and Preliminaries of Fractional-Order Calculus}

Fractional-order integration and differentiation are the generalization of the integer-order ones. Efforts to extend the specific definitions of the traditional integer-order to the more general arbitrary order context led to different definitions for fractional derivatives [4]. Riemann-Liouville definition is adopted in this paper.
Definition 1 (see [2]). The $\alpha$ th-order Riemann-Liouville fractional derivative of function $f(t)$ with respect to $t_{0}$ and $t$ is given by

$$
D^{\alpha} f(t)=\frac{1}{\Gamma(m-\alpha)} \frac{d^{m}}{d t^{m}} \int_{t_{0}}^{t} \frac{f(\tau)}{(t-\tau)^{\alpha-m+1}} d \tau,
$$

and the Riemann-Liouville definition of the $\alpha$ th-order fractional integration is given by

$$
{ }_{t_{0}} I_{t}^{\alpha} f(t)=\frac{1}{\Gamma(\alpha)} \int_{t_{0}}^{t} \frac{f(\tau)}{(t-\tau)^{1-\alpha}} d \tau
$$

where $m-1 \leq \alpha<m, m$ is the first integer larger than $\alpha$, and $\Gamma$ is the Gamma function.

Lemma 2 (see [2]). If the Riemann-Liouville fractional derivative ${ }_{a} D_{t}^{\alpha} f(t)(n-1 \leq \alpha<n)$ of a function $f(t)$ is integrable, then

$$
\begin{aligned}
{ }_{a} D_{t}^{-\alpha}\left({ }_{a} D_{t}^{\beta} f(t)\right)= & { }_{a} D_{t}^{\beta-\alpha} f(t) \\
& -\sum_{j=1}^{n}\left[{ }_{a} D_{t}^{\beta-j} f(t)\right]_{t=a} \frac{(t-a)^{\alpha-j}}{\Gamma(1+\alpha-j)} .
\end{aligned}
$$

Lemma 3 (see [30]). Fractional derivatives and integrals have the following properties, namely,

Semigroup Property:

$$
{ }_{a} D_{t}^{-\alpha}{ }_{a} D_{t}^{-\beta} f(t)={ }_{a} D_{t}^{-\alpha-\beta} f(t) .
$$

Composition Rules:

$$
\begin{gathered}
\frac{d^{n}}{d x^{n}}\left[{ }_{a} D_{t}^{\alpha} f(t)\right]={ }_{a} D_{t}^{n+\alpha} f(t) \\
{ }_{a} D_{t}^{\alpha}\left[\frac{d^{n}}{d x^{n}} f(t)\right]={ }_{a} D_{t}^{n+\alpha} f(t)-\sum_{j=0}^{n-1} \frac{f^{(j)}(a)(t-a)^{j-\alpha-n}}{\Gamma(1+j-\alpha-n)}
\end{gathered}
$$

where $\alpha>0, t \geq a, \beta>0$, and $n-1 \leq \alpha<n$.

Lemma 4 (see [4]). The fractional integration operator ${ }_{a} I_{t}^{\alpha}$ with $\alpha>0$ is bounded,

$$
\left\|I^{\alpha} y\right\| \leq K\|y\|_{p}, \quad 0 \leq K<\infty, 1 \leq p \leq \infty .
$$

\section{Problem Statement and Preliminaries}

Consider the following nonlinear systems

$$
\ddot{x}=F(x, t)+G(x, t) u(t)+d(x, t),
$$

where $x=\left[x_{1}, x_{2}, \ldots, x_{n}\right]^{T}, u(t)$ is the control effort, $d(x, t)$ is the external disturbance, $F(x, t)=F_{n}(x, t)+$ $\Delta F(x, t), \Delta F(x, t)$ is an uncertain term representing the unmodeled dynamics or structural variation of the system, $G(x, t)=G_{n}(x, t)+\Delta G(x, t)$, and $\Delta G(x, t)$ is the input uncertainty. In our study of this nonlinear system, we will impose one assumption. 
Assumption. The lumped uncertainty $L(x, t)$ is defined as

$$
L(x, t)=\Delta F(x, t)+\Delta G(x, t) u(t)+d(x, t),
$$

where $L(x, t)$ satisfies the condition that $\left\|D^{1+\alpha}(L(x, t))\right\| \leq \delta$ and $\delta$ is a given positive constant.

Remark 5. It is needed to emphasis that this hypothesis is not restrictive as it can be satisfied by many dynamical systems in the literature [29], such as inverted pendulum, chaotic gyro [31], magnetic bearing [32, 33], and ship roll motion [34]. We will remove this assumption later in Section 6.

The tracking error can be defined as

$$
e(t)=x_{d}(t)-x(t) .
$$

The control objective considered in this paper is to design a robust fractional-order terminal sliding mode controller to stabilize state variables in finite time. In the next section, we will discuss how to design the appropriate switching surface and the fractional-order fast terminal sliding mode controller.

\section{Review of Fractional-Order Terminal Sliding Mode Control (FO-TSMC)}

Theorem 6 (see [29]). Let the sliding surface be defined as

$$
s=D^{\alpha} \ddot{e}+\lambda e(t)^{p / q}, \quad \alpha \in(0,1),
$$

where $p$ and $q$ are positive odd integers. Consider the system (8) in which the lumped uncertainties satisfy the constraint that $\left\|D^{\alpha+1}(L(x, t))\right\| \leq \delta$. If the terminal sliding mode control law is designed as follows:

$$
\begin{aligned}
u(t)=G_{n}^{-1}[ & \ddot{x}_{d}(t)-F_{n}(x, t)+D^{-1-\alpha} \\
& \left.\times\left(\lambda \frac{p}{q} e^{(p / q)-1} \dot{e}+k s+K \operatorname{sgn}(s)\right)+\delta\right],
\end{aligned}
$$

where $k$ and $K$ are positive constants, $\operatorname{sgn}(s)$ is the signum function of sliding surfaces, and $\lambda$ is a positive design constant, then the tracking error converges to zero in finite time.

The derivative of (11) with respect to time can be represented by

$$
\dot{s}=D^{1+\alpha} \ddot{e}+\lambda \frac{p}{q} e(t)^{(p / q)-1} \dot{e} .
$$

Remark 7. In this paper, to eliminate the chattering, the control input is modified as

$$
\begin{aligned}
u(t)=G_{n}^{-1}[ & \ddot{x}_{d}(t)-F_{n}(x, t) \\
& \left.+D^{-1-\alpha}\left(\lambda \frac{p}{q} e^{(p / q)-1} \dot{e}+k s+K \operatorname{sig}^{\rho}(s)\right)+\delta\right],
\end{aligned}
$$

where $\rho$ is a positive design parameter, $\operatorname{sig}^{\rho}(s)=|s|^{\rho} \operatorname{sgn}(s)$.

\section{Fractional Order Fast Terminal Sliding- Mode Control (FO-FTSMC)}

The fractional-order sliding surface can be defined as follows:

$$
s=D^{\alpha} \ddot{e}+k_{1} \operatorname{sig}^{a_{1}}(e)+k_{2} \operatorname{sig}^{a_{2}}(e),
$$

where $0 \leq \alpha<1, k_{1}=\operatorname{diag}\left(k_{11}, \ldots, k_{1 n}\right)>0, k_{2}=$ $\operatorname{diag}\left(k_{21}, \ldots, k_{2 n}\right)>0, \operatorname{sig}^{a_{i}}(e)=|e|^{a_{i}} \operatorname{sgn}(e)(i=1,2), a_{1}>1$, and $0<a_{2}=p / q<1$, where $p$ and $q$ are odd numbers. Note that

$$
\frac{d}{d t} \operatorname{sig}^{a_{i}}(e)=a_{i}|e|^{a_{i}-1} \dot{e}=a_{i} \operatorname{sig}^{a_{i}-1}(e) \operatorname{sgn}(e) \dot{e} .
$$

A fast terminal sliding mode type reaching law is defined as

$$
\dot{s}=D^{1+\alpha} \ddot{e}+\left(\bar{k}_{1}|e|^{a_{1}-1}+\bar{k}_{2}|e|^{a_{2}-1}\right) \dot{e},
$$

where $\bar{k}_{1}=k_{1} a_{1}$ and $\bar{k}_{2}=k_{2} a_{2}$. If the terminal mode control law is designed as follows:

$$
\begin{aligned}
u=G_{n}^{-1}\left[\ddot{x}_{d}(t)+D^{-\alpha-1}\left(\left(\bar{k}_{1}|e|^{a_{1}-1}+\bar{k}_{2}|e|^{a_{2}-1}\right) \dot{e}\right)\right. \\
\left.\quad-F_{n}(x, t)+D^{-\alpha-1}\left(\gamma_{1} s+\gamma_{2} \operatorname{sig}^{\rho}(s)+\delta \operatorname{sgn}(s)\right)\right],
\end{aligned}
$$

where $0<\rho<1$, the following theorem holds.

Theorem 8. Let the sliding surface be defined by (15), where $p$ and $q$ are positive odd integers. Consider the system (8) in which the lumped uncertainties satisfy the constraint that $\left\|D^{\alpha+1}(L(x, t))\right\| \leq \delta$. If the terminal sliding mode control law is set as (18), the tracking error converges to zero in finite time.

Proof. Let the Lyapunov function be defined as

$$
V=\frac{1}{2} s^{T} s .
$$

Taking derivative of (19) with respect to time, using (5), (18), and $f(t)=D^{1+\alpha}\left(D^{-(1+\alpha)} f(t)\right)$, we have

$$
\begin{aligned}
\dot{V}= & s^{T} \dot{s}=s^{T}\left(D^{1+\alpha} \ddot{e}+\left(\bar{k}_{1}|e|^{a_{1}-1}+\bar{k}_{2}|e|^{a_{2}-1}\right) \dot{e}\right) \\
= & s^{T}\left(D^{1+\alpha}\left(\ddot{x}_{d}(t)-F_{n}(x, t)-G_{n}(x, t) u(t)-L(x, t)\right)\right. \\
& \left.\quad+\left(\bar{k}_{1}|e|^{a_{1}-1}+\bar{k}_{2}|e|^{a_{2}-1}\right) \dot{e}\right) \\
= & s^{T}\left(-D^{1+\alpha}\left(D^{-\alpha-1}\left(\left(\bar{k}_{1}|e|^{a_{1}-1}+\bar{k}_{2}|e|^{a_{2}-1}\right) \dot{e}\right)\right)\right. \\
& \quad-D^{1+\alpha}\left(D^{-\alpha-1}\left(\gamma_{1} s+\gamma_{2} \operatorname{sig}^{\rho}(s)+\delta \operatorname{sgn}(s)\right)\right) \\
= & \left.\quad-D^{1+\alpha}(L(x, t))+\left(\bar{k}_{1}|e|^{a_{1}-1}+\bar{k}_{2}|e|^{a_{2}-1}\right) \dot{e}\right) \\
= & s^{T}\left(-\gamma_{1} s-\gamma_{2} \operatorname{sig}^{\rho}(s)-\delta \operatorname{sgn}(s)-D^{1+\alpha}(L(x, t))\right)-\delta|s|-s^{T} D^{1+\alpha}(L(x, t)) \\
\leq & s^{T}\left(-\gamma_{1} s-\gamma_{2} \operatorname{sig}^{\rho}(s)\right)=-\gamma_{1}\|s\|^{2}-\gamma_{2}\|s\|^{\rho+1} .
\end{aligned}
$$


Choosing $\gamma_{1}>0$ and $\gamma_{2}>0$ appropriately, we can obtain $\dot{V}<0$ when $\|s\| \neq 0$, so the sliding motion converges to zero. In order to verify that the tracking error occurs in finite time, we can define a stopping time as follows:

$$
\tau_{s}=\inf \left\{t \geq t_{r} \mid e(t)=0\right\},
$$

where $t_{r}$ is the time for the system states to reach the sliding surface and $s\left(t_{r}\right)=0$. Taking the concept of fractional integral and derivative operators into (15), we have

$$
D^{-\alpha}\left(D^{\alpha} \ddot{e}(t)\right)=D^{-\alpha}\left(-k_{1} \operatorname{sig}^{a_{1}} e-k_{2} \operatorname{sig}^{a_{2}} e\right) .
$$

By Lemma 2, the following holds:

$$
\begin{aligned}
\ddot{e}(t) & -\left[{ }_{t_{r}} D_{t}^{\alpha-1} \ddot{e}(t)\right]_{t=t_{r}} \frac{\left(t-t_{r}\right)^{\alpha-1}}{\Gamma(\alpha)} \\
= & -D^{-\alpha}\left(k_{1} \operatorname{sig}^{a_{1}} e+k_{2} \operatorname{sig}^{a_{2}} e\right) .
\end{aligned}
$$

Since $\alpha-1<0$, it follows that

$$
\left[t_{r} D_{t}^{\alpha-1} \ddot{e}(t)\right]_{t=t_{r}} \frac{\left(t-t_{r}\right)^{\alpha-1}}{\Gamma(\alpha)}=0 .
$$

Combining (23) and (24), we have

$$
\ddot{e}(t)=-D^{-\alpha}\left(k_{1} \operatorname{sig}^{a_{1}} e+k_{2} \operatorname{sig}^{a_{2}} e\right) .
$$

By the Riemann-Liouville definition, Lemma 2, operator ${ }_{t_{r}} D_{t}^{-2}$, and the associativity law, we deduce

$$
\begin{aligned}
e(t) & -\left[{ }_{t_{r}} D_{t} e(t)\right]_{t=t_{r}} \frac{\left(t-t_{r}\right)}{2}-e\left(t_{r}\right) \\
& =-{ }_{t_{r}} D_{t}^{-2-\alpha}\left(k_{1} \operatorname{sig}^{a_{1}} e+k_{2} \operatorname{sig}^{a_{2}} e\right) .
\end{aligned}
$$

According to Lemma 4, we have

$$
\begin{gathered}
{ }_{t_{r}} D_{t}^{-2-\alpha}\left(k_{1} \operatorname{sig}^{a_{1}} e+k_{2} \operatorname{sig}^{a_{2}} e\right) \\
\leq K_{1}\left\|e^{a_{1}}\right\|+K_{2}\left\|e^{a_{2}}\right\|,
\end{gathered}
$$

where $K_{1}=K k_{1}$ and $K_{2}=K k_{2}$. It follows that

$$
\begin{aligned}
\| e(t) & -\left[{ }_{t_{r}} D_{t} e(t)\right]_{t=t_{r}} \frac{\left(t-t_{r}\right)}{2} \| \\
& -\left\|e\left(t_{r}\right)\right\| \leq K_{1}\left\|e^{a_{1}}\right\|+K_{2}\left\|e^{a_{2}}\right\| .
\end{aligned}
$$

Noting that $e\left(t_{s}\right)=0$, it yields

$$
\left\|\frac{\left[{ }_{t_{r}} D_{t} e(t)\right]_{t=t_{r}}}{2}\right\|\left(t_{s}-t_{r}\right) \leq\left\|e\left(t_{r}\right)\right\| .
$$

We will study (29) in two cases, if $\left\|\dot{e}\left(t_{r}\right)\right\| \neq 0$, it yields

$$
t_{s} \leq \frac{2\left\|e\left(t_{r}\right)\right\|}{\left\|\dot{e}\left(t_{r}\right)\right\|}+t_{r} .
$$

If $\left\|\dot{e}\left(t_{r}\right)\right\|=0, e\left(t_{r}\right)=0$, we have $t_{s}=t_{r}$. In the following, consider the case that $e\left(t_{r}\right) \neq 0$. We know that $\left\|\dot{e}\left(t_{r}\right)\right\|=$ $0,\|e(t)\| \leq\left\|e\left(t_{r}\right)\right\|$. For $e(t)<0$, we have $e(t) \rightarrow 0$ as $t \rightarrow \infty$; for $e(t)>0$, we have $e(t) \rightarrow 0$ as $t \rightarrow \infty$. For simplicity, we just consider the case $\left\|\dot{e}\left(t_{r}\right)\right\|=0, e\left(t_{r}\right)>0$, and $t \geq t_{r}$. The proof of another case is similar. By composition rules of Lemma 3 and (15), we have

$$
\begin{aligned}
D^{\alpha} \ddot{e}(t) & =D^{1+\alpha} \dot{e}(t)-\frac{\dot{e}\left(t_{r}\right)\left(t-t_{r}\right)^{-\alpha-n}}{\Gamma(1-\alpha-n)} \\
& =D^{1+\alpha} \dot{e}(t)=-k_{1} \operatorname{sig}^{a_{1}} e-k_{2} \operatorname{sig}^{a_{2}} e \\
& \leq-k_{1} \operatorname{sig}^{a_{1}} e=-k_{1} e^{a_{1}} .
\end{aligned}
$$

Since $\left\|\dot{e}\left(t_{r}\right)\right\|=0$, it follows that

$$
\begin{aligned}
& \sum_{j=1}^{2}\left[{ }_{r} D_{t}^{\alpha+1-j} \dot{e}(t)\right]_{t=t_{r}} \frac{\left(t-t_{r}\right)^{\alpha+1-j}}{\Gamma(2-j)}=0, \\
& D^{-(\alpha+1)}\left(D^{1+\alpha} \dot{e}(t)\right) \\
&=\dot{e}(t)-\sum_{j=1}^{2}\left[{ }_{t_{r}} D_{t}^{\alpha+1-j} \dot{e}(t)\right]_{t=t_{r}} \frac{\left(t-t_{r}\right)^{\alpha+1-j}}{\Gamma(2-j)}=\dot{e}(t) .
\end{aligned}
$$

Based on $\left\|\dot{e}\left(t_{r}\right)\right\|=0$ and $e\left(t_{r}\right)>0$, by Definition 1 , we have

$$
\begin{aligned}
& D^{-(\alpha+1)}\left(-k_{1} e^{a_{1}}\right) \\
& \quad=\frac{-k_{1}}{\Gamma(\alpha+1)} \int_{t_{r}}^{t} \frac{e^{a_{1}}(\tau)}{(t-\tau)^{\alpha}} d \tau \leq \frac{-k_{1}}{\Gamma(\alpha+1)} \int_{t_{r}}^{t} \frac{e^{a_{1}}(\tau)}{\left(t_{s}-t_{r}\right)^{\alpha}} d \tau,
\end{aligned}
$$

where $0<(t-\tau)^{\alpha} \leq\left(t_{s}-t_{r}\right)^{\alpha}$ whenever $t \geq t_{r}$. Thus, (33) can be simplified as

$$
\dot{e}(t) \leq \frac{-k_{1}}{\Gamma(\alpha+1)} \int_{t_{r}}^{t} \frac{e^{a_{1}}(\tau)}{\left(t_{s}-t_{r}\right)^{\alpha}} d \tau,
$$

integrating (35) with respect to $t$ on interval $\left[t_{r}, t_{s}\right]$ and combining with $e\left(t_{s}\right)=0$ and (34), the following inequality holds:

$$
-e\left(t_{r}\right) \leq \frac{-k_{1}}{\Gamma(\alpha+1)}\left(t_{s}-t_{r}\right)^{-\alpha} \int_{t_{r}}^{t_{s}} \int_{t_{r}}^{t} e^{a_{1}}(\tau) d \tau d t .
$$

For the double integral in (36), we apply the mean value theorem, there exist $\xi_{1} \in\left(t_{r}, t_{s}\right)$ and $e\left(\xi_{1}\right)>0$, satisfy

$$
-e\left(t_{r}\right) \leq \frac{-k_{1} e^{a_{1}}\left(\xi_{1}\right)\left(t_{s}-t_{r}\right)^{2-\alpha}}{2 \Gamma(\alpha+1)} .
$$


Finally, we get

$$
t_{s} \leq t_{r}+\left(\frac{2 \Gamma(\alpha+1) e\left(t_{r}\right)}{k_{1} \cdot e^{a_{1}}\left(\xi_{1}\right)}\right)^{1 /(2-\alpha)}
$$

From (38) we can see that $t_{s}$ cannot be infinity; actually, the right hand of (38) is finite when $t_{r}<t<t_{s}$. It can be concluded that the system trajectories attain to reference trajectories in finite time.

Remark 9. The fractional sliding surface (15) is slightly different from the FTSMC [24] which is expressed as

$$
s=\dot{e}+k_{1} \operatorname{sig}^{a_{1}}(e)+k_{2} \operatorname{sig}^{a_{2}}(e),
$$

where $k_{1}>0, k_{2}>0, a_{1}>1$, and $0<a_{2}<1$. Note that $\operatorname{sig}^{a_{i}} e=|e|^{a_{i}} \operatorname{sgn}(e)(i=1,2)$. Letting $a_{2}=p / q$, the fractionalorder fast terminal sliding-mode control (FO-FTSMC) in (15) does not have the singularity problem except $e(t)=0$.

\section{Fractional-Order Nonsingular Fast Terminal Sliding-Mode Control}

During the control design of TSMs, there often exists singularity problem. The reason is that the designed controllers often have nonlinear terms, such as $x^{-\alpha}$, which results in the unboundedness of the control input as $x$ approaches to zero. In the controller design, a nonsingular item $|\dot{e}|^{2-a_{2}}$ is adopted to avoid this problem. To achieve equivalence between convergence rate and singularity avoidance, fractional-order nonsingular fast terminal slidingmode control (FO-NFTSMC) is proposed, and the method is described as

$$
s=D^{\alpha} e+k_{1} \operatorname{sig}^{a_{1}} e+k_{2} \operatorname{sig}^{a_{2}} \dot{e}=0,
$$

where $k_{1}>0, k_{2}>0,1<a_{2}<2$, and $a_{1}>a_{2}$. We choose the terminal sliding mode control law as

$$
\begin{aligned}
u=G_{n}^{-1}\left[\ddot{x}_{d}-F_{n}(x, t)+\bar{k}_{2}^{-1}|\dot{e}|^{2-a_{2}}\right. \\
\cdot\left(\gamma_{1} s+\gamma_{2} \operatorname{sig}^{\rho} s\right)+\delta \operatorname{sgn}(s)+\bar{k}_{2}^{-1}|\dot{e}|^{2-a_{2}} \\
\left.\quad \times\left(D^{1+\alpha} e+\bar{k}_{1}|e|^{a_{1}-1} \cdot \dot{e}\right)\right],
\end{aligned}
$$

where $\bar{k}_{1}=k_{1} a_{1}, \bar{k}_{2}=k_{2} a_{2}, \bar{k}_{2}^{-1}=1 / \bar{k}_{2}$, and $\gamma_{1}, \gamma_{2}$ are the design parameters.

Theorem 10. Let the sliding surface be defined by (40), where $k_{1}>0, k_{2}>0,1<a_{2}<2$, and $a_{1}>a_{2}$. Consider the system (8) in which the lumped uncertainties satisfy the constraint that $\|L(x, t)\| \leq \delta$. If the terminal sliding mode control law is set as (41), the tracking error converges to zero in finite time.
Proof. Let the Lyapunov function be defined as (19). Taking derivative of (19) with respect to time and using (41) yields

$$
\begin{aligned}
\dot{V}= & s^{T} \dot{s}=s^{T}\left(D^{1+\alpha} e+\bar{k}_{1}|e|^{a_{1}-1} \cdot \dot{e}+\bar{k}_{2}|\dot{e}|^{a_{2}-1} \cdot \ddot{e}\right) \\
= & s^{T}\left(\overline { k } _ { 2 } | \dot { e } | ^ { a _ { 2 } - 1 } \cdot \left(\ddot{x}_{d}(t)-F_{n}(x, t)\right.\right. \\
& \left.\left.\quad-G_{n}(x, t) u(t)-L(x, t)\right)\right) \\
& +s^{T}\left(D^{1+\alpha} e+\bar{k}_{1}|e|^{a_{1}-1} \cdot \dot{e}\right) \\
= & s^{T} \bar{k}_{2}|\dot{e}|^{a_{2}-1} \cdot(-\delta \operatorname{sgn}(s)-L(x, t)) \\
& +s^{T}|\dot{e}|\left(-\gamma_{1} s-\gamma_{2} \operatorname{sig} g^{\rho}(s)\right)-s^{T}(|\dot{e}|-1) \\
& \times\left(D^{1+\alpha} e+\bar{k}_{1}|e|^{a_{1}-1} \cdot \dot{e}\right) \\
\leq & -s^{T}|\dot{e}|\left(\gamma_{12} s+\gamma_{22} \operatorname{sig}^{\rho}(s)\right) \\
= & -|\dot{e}|\left(\gamma_{12}\|s\|^{2}+\gamma_{22}\|s\|^{\rho+1}\right),
\end{aligned}
$$

where $\gamma_{1}=\gamma_{11}+\gamma_{12}, \gamma_{2}=\gamma_{21}+\gamma_{22}, \gamma_{11}>0, \gamma_{12}>0, \gamma_{21}>$ $0, \gamma_{22}>0$, and $s^{T}|\dot{e}|\left(-\gamma_{11} s-\gamma_{21} \operatorname{sig}^{\rho}(s)\right)-s^{T}(|\dot{e}|-1)\left(D^{1+\alpha} e+\right.$ $\left.\bar{k}_{1}|e|^{a_{1}-1} \cdot \dot{e}\right)<0$ when $\gamma_{1}$ and $\gamma_{2}$ are appropriately chosen. Thus, we can verify that $\dot{V}<0$ as $\|s\| \neq 0, \dot{e}(t) \neq 0$. The finite time reach ability will be shown by simulation. The proof is completed.

Remark 11. Equation (40) is equivalent to Yang's NFTSM [24] when $\alpha=0, D^{\alpha} e=e$. For $1<a_{2}<2$ and $a_{1}>a_{2}$, we can verify that no singularity problem occurred in (41). Furthermore, $\left\|D^{\alpha+1}(L(x, t))\right\| \leq \delta$ is replaced by $\|L(x, t)\| \leq \delta$ which is easy to be satisfied in practice.

Remark 12. The sliding surface proposed by Liang is defined as $s=e+k_{1} \operatorname{sig}^{a_{1}} e+k_{2} \operatorname{sig}^{a_{2}} \dot{e}=0$ [24]; we replace the error $e$ by $D^{\alpha} e$; thus, (40) is obtained. We can see that the new sliding surface can perform better if appropriate design parameters are chosen.

\section{Simulation Results}

In this section, we will give three illustrative examples to show the applicability and efficiency of the proposed controller. The first example is a magnetic bearing system, the second one is a single inverted pendulum, and the last one is a two-link manipulator.

7.1. Magnetic Bearing System. Consider the uncertain magnetic bearing system $[29,33]$ with the following form:

$$
m \ddot{x}(t)=-c \dot{x}+k_{x} x+k_{i} i_{x}+d(x, t),
$$

where $m$ is the mass of rotor, $c$ is the friction, and $k_{x}$ and $k_{i}$ are the position and current stiffness, respectively; $d(x, t)$ is the external disturbance and $i_{x}$ is control current (control input). Moreover, the variable state $x$ denotes the deviation of the nominal air gap which is also referred as the rotor position in the magnetic bearing system. 

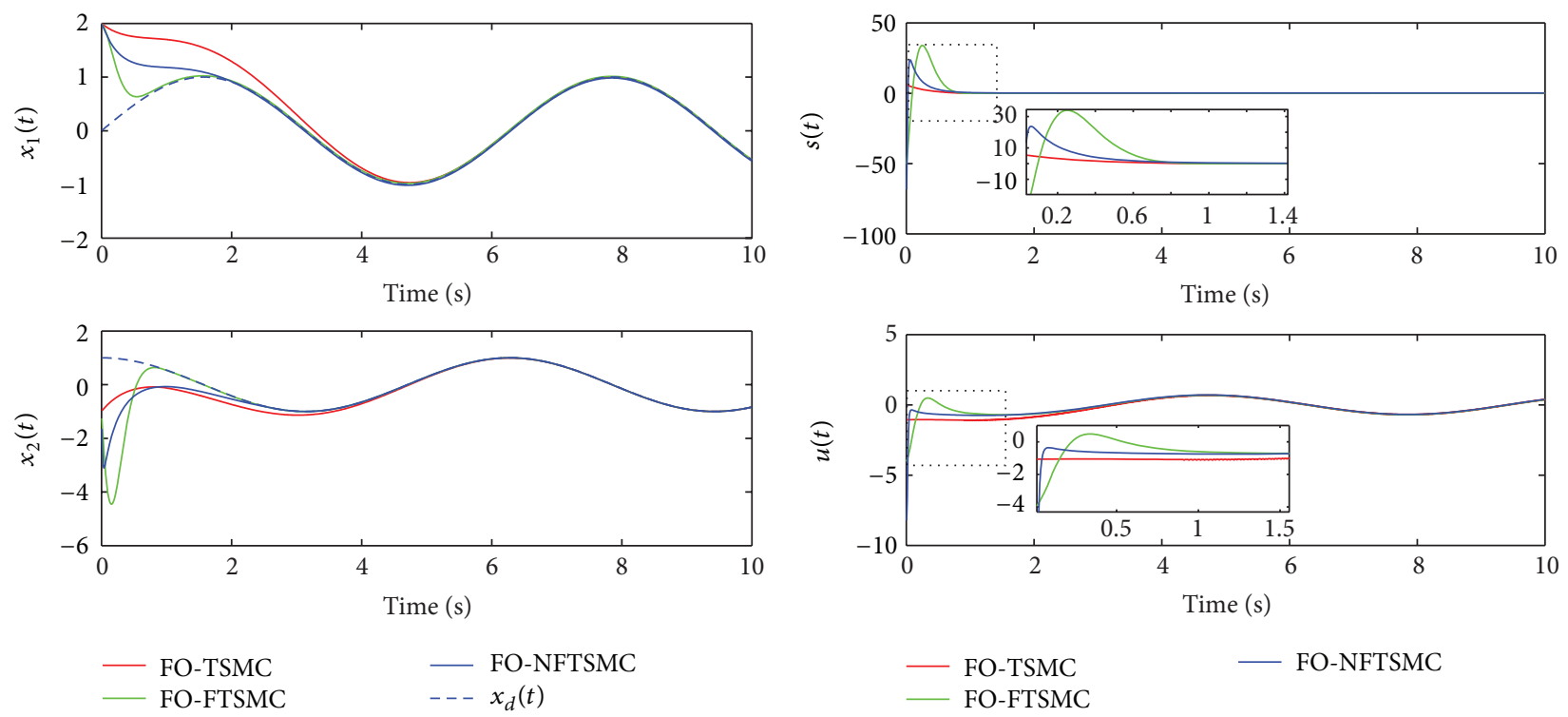

(a)

(b)

FIGURE 1: (a) The time response of the magnetic bearing system states $\left(x_{d}=\sin (t)\right)$. (b) The time response of sliding surface and control input for magnetic bearing system states $\left(x_{d}=\sin (t)\right)$.

The following parameters are employed in the numerical simulations: $m=2.565 \mathrm{~kg}, c=0.001, k_{x}=25.2, k_{i}=$ $40, \alpha=0.9$, and $x_{0}=[2,-5]^{T}$. The parameters for FO-TSMC are defined as $p=23, q=25, k=1, K=5, \lambda=1$, and $\rho=1$. The parameters for FO-FTSMC are defined as $k_{1}=10, k_{2}=10, a_{1}=2, a_{2}=3 / 5, \gamma_{1}=10, \gamma_{2}=10$, and $\rho=2 / 5$. The parameters for FO-NFTSMC are defined as $k_{1}=15, k_{2}=6, a_{1}=3, a_{2}=5 / 3, \gamma_{1}=15, \gamma_{2}=6$, and $\rho=2 / 5$.

State responses of the closed loop system are shown in Figure 1. Figure 1(a) represents the state time response for tracking a desired trajectory $x_{d}=\sin (t)$. It can be seen that the dynamics of system states are stabilized to a given motion. The corresponding $s(t)$ and control input $u(t)$ are illustrated in Figure 1(b). We can infer that, among the three fractional-order controllers, FO-FTSMC can track the reference signal in the shortest time, while the control input is slightly larger than FO-NFTSMC and FO-TSMC. The time taken by FO-NFTSMC is shorter than FO-TSMC, while the control input is slightly smaller than FO-NFTSMC and FO-TSMC.

Figure 2(a) shows the state responses for regulating the states to $x_{d}=0$. Time response of sliding surface and the control effort of the three controllers are presented in Figure 2(b). Results show that the trajectories of FO-FTSMC and FO-NFTSMC converge faster than that of FO-TSMC. The boundary layer for FO-TSMC is $\|s\| \leq 2 \times 10^{-2}$, while FO-FTSMC and FO-NFTSMC keep the boundary layer with $2 \times 10^{-3}$ and $2 \times 10^{-2}$.

7.2. Single Inverted Pendulum. The problem to be considered is a pole-balancing of an inverted pendulum. The system is represented by

$$
\begin{gathered}
\dot{x}_{1}=x_{2}, \\
\dot{x}_{2}=\frac{m l x_{2}^{2} \sin x_{1} \cos x_{1}-(M+m) g \sin x_{1}}{m l \cos ^{2}\left(x_{1}\right)-4 / 3 l(M+m)} \\
-\frac{\cos x_{1}}{m l \cos ^{2}\left(x_{1}\right)-4 / 3 l(M+m)} u,
\end{gathered}
$$

where $x_{1}$ is the angle (in radians) of the pendulum from the vertical, $M$ is the mass of the cart, $m$ is the mass of the pole, $g$ is the gravitational constant, $l$ is the half length of the pole, and $u$ is the force applied to the cart. The parameters employed in this simulation of inverted pendulum are given as follows: $M=1 \mathrm{~kg}, m=0.1 \mathrm{~kg}, l=0.5 \mathrm{~m}$, and $g=9.8 \mathrm{~m} / \mathrm{s}^{2}$. In order to eliminate the chattering, a modified reaching law $k_{1} s+K_{2} \operatorname{sig}^{\rho}(s)$ is used for FO-TSMC. The parameters for FOTSMC are defined as $p=3, q=5, K_{1}=15, K_{2}=3, \lambda=$ $10, \alpha=0.9$, and $\rho=4 / 5$. The parameters for FO-FTSMC are defined as $k_{1}=10, k_{2}=10, a_{1}=2, a_{2}=3 / 5, \alpha=0.9, \gamma_{1}=$ 20, $\gamma_{2}=20$, and $\rho=4 / 5$. The parameters for FO-NFTSMC are defined as $k_{1}=3, k_{2}=3, a_{1}=2, a_{2}=5 / 3, \alpha=0.9, \gamma_{1}=$ $3, \gamma_{2}=3$, and $\rho=3 / 5$. Figures 3(a) and 3(b) show that the controller can track the trajectories well and the chattering of the FO-TSMC is eliminated.

7.3. Two-Link Manipulator System. A two-link rigid robotic manipulator is used to evaluate the performance of the proposed adaptive FO-FTSMC and FO-NFTSMC. The dynamic equation of the robotic manipulator is given by Feng et al. [28] as

$$
M(q) \ddot{q}+C(q, \dot{q})+G(q)=\tau(t)+\tau_{d},
$$



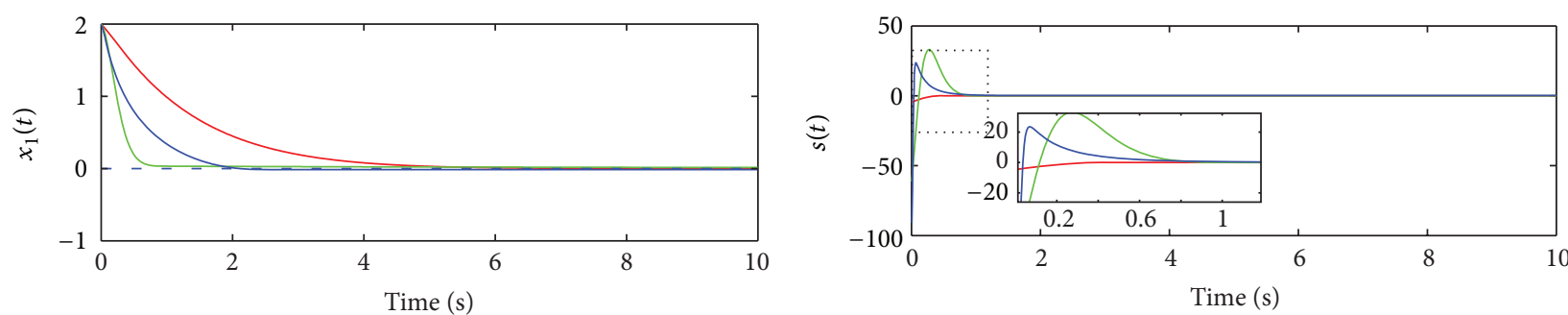

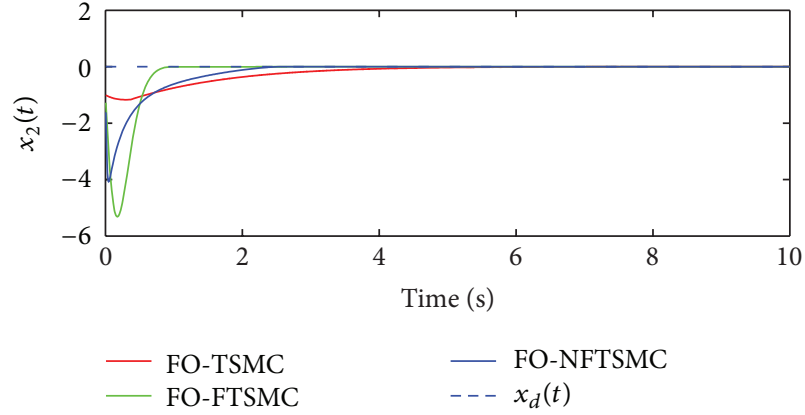

(a)

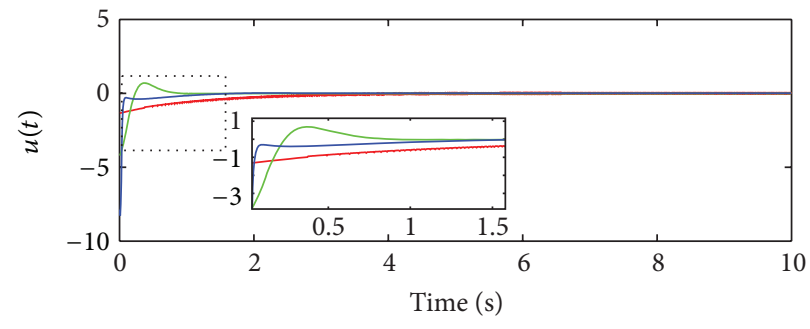

FO-TSMC

FO-FTSMC

(b)

Figure 2: (a) The time response of the magnetic bearing system states $\left(x_{d}=0\right)$. (b) The time response of sliding surface and control input for magnetic bearing system states $\left(x_{d}=0\right)$.
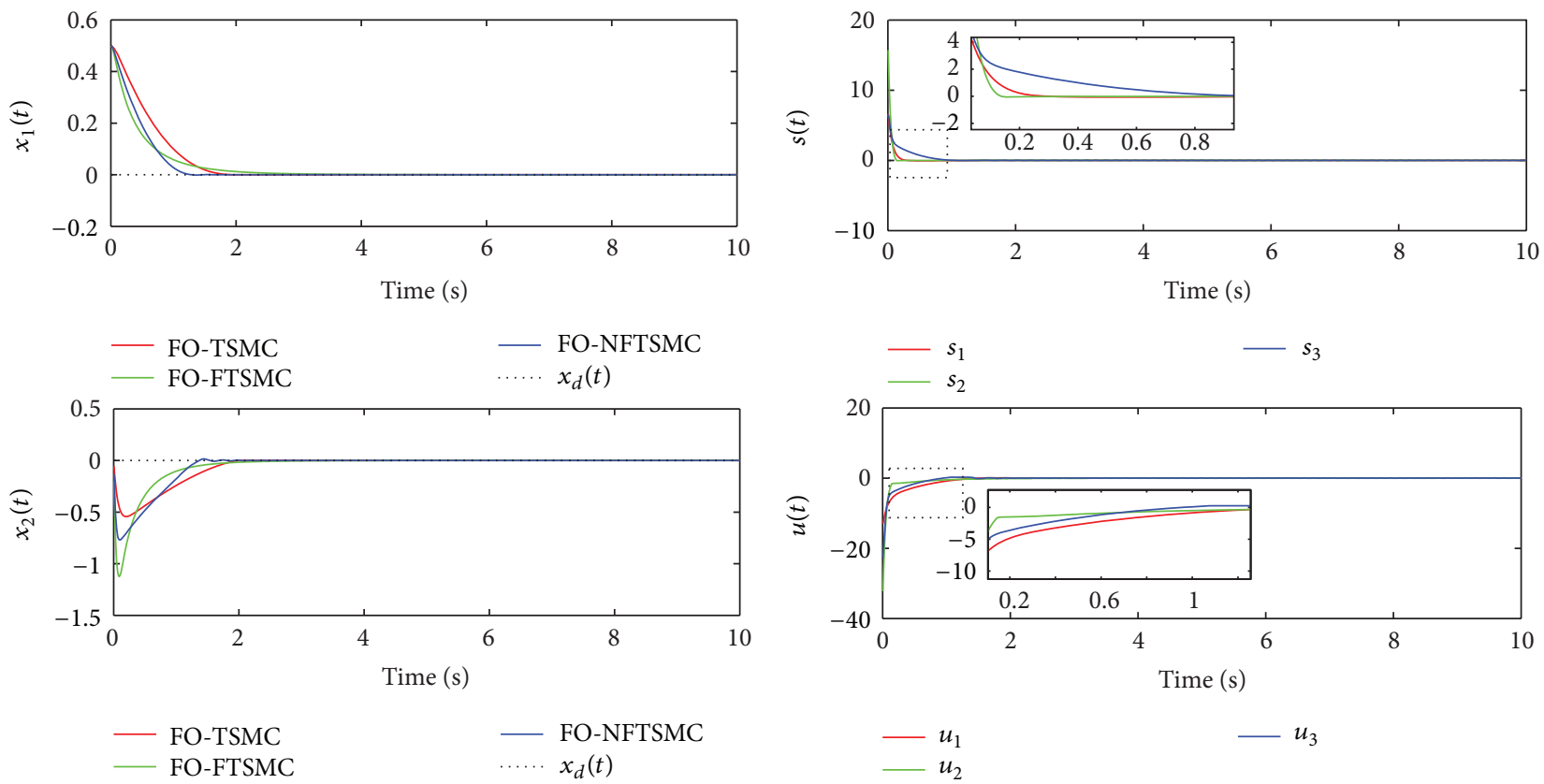

(a)

(b)

FIGURE 3: (a) The time response of the single inverted pendulum states $\left(x_{d}=0\right)$. (b) The time response of sliding surface and control input for single inverted pendulum system states $\left(x_{d}=0\right)$. 

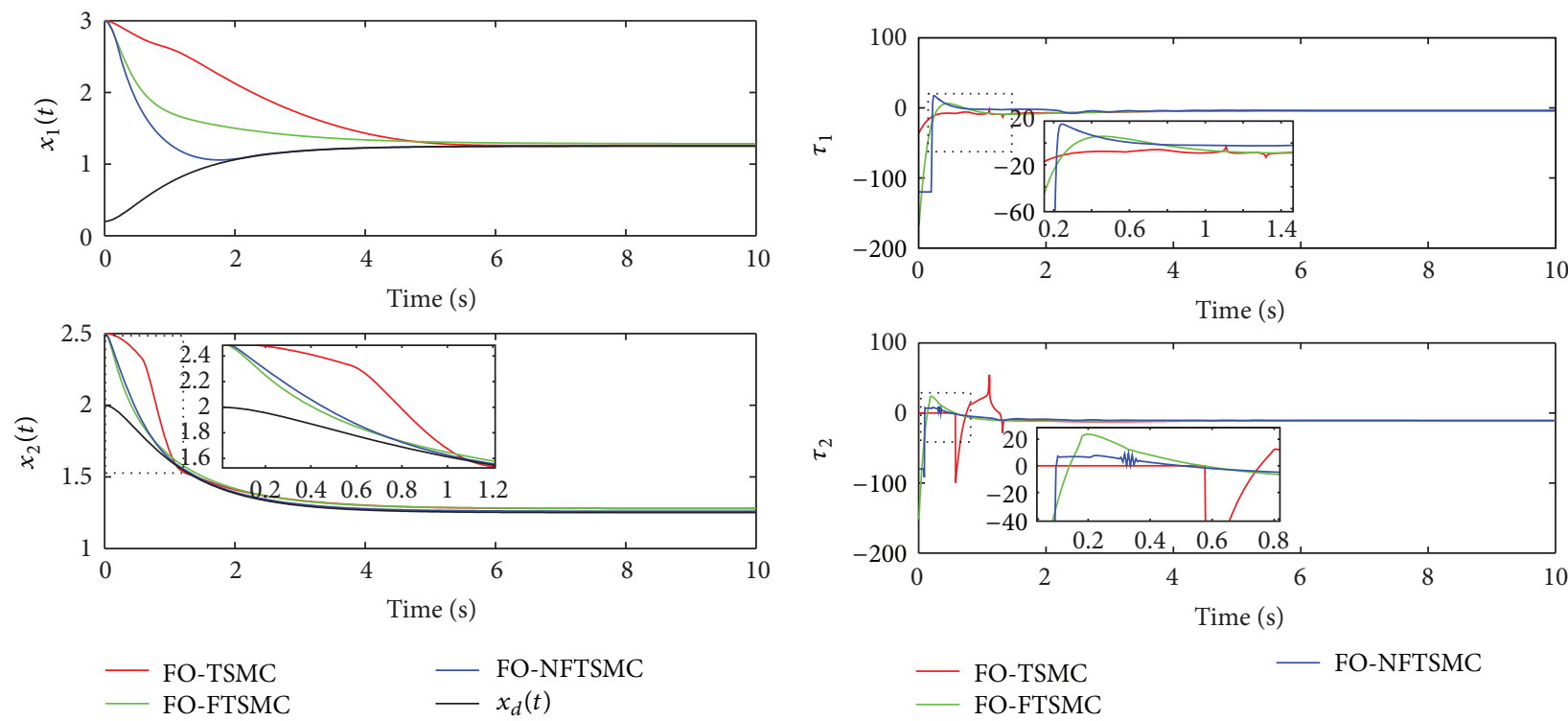

(a)

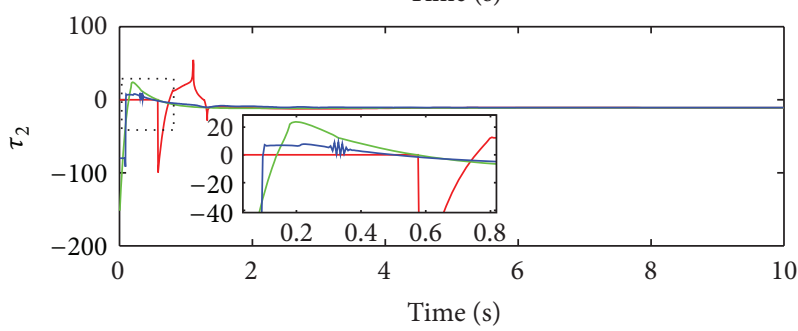

FO-TSMC
- FO-FTSMC

(b)

FIgURE 4: (a) The time response of the two-link manipulator system states. (b) The control input for two-link manipulator system states.
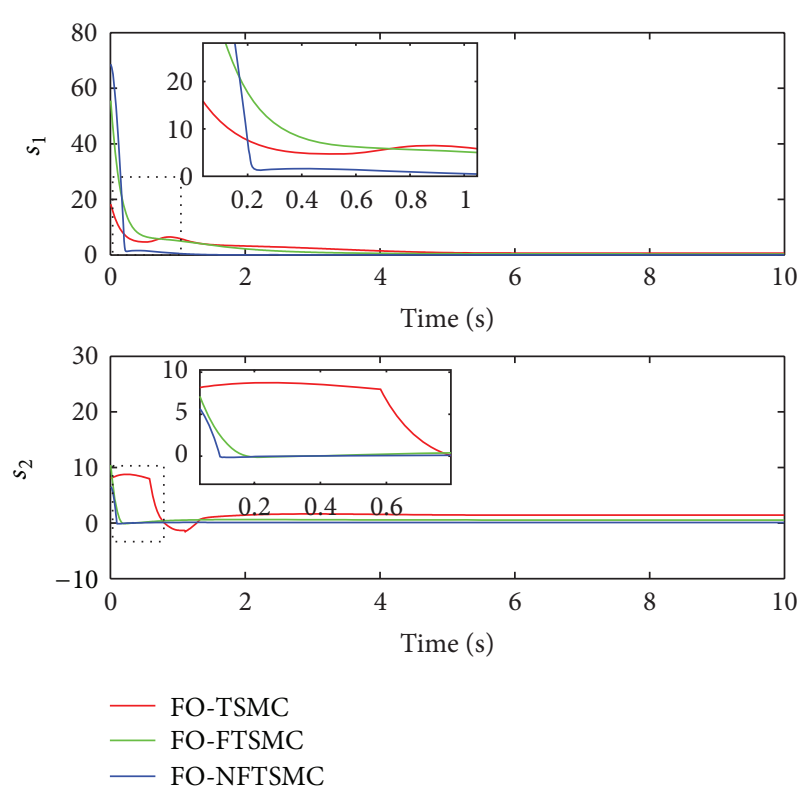

FIGURE 5: The time response of sliding surface for two-link manipulator system.

where $q, \dot{q}, \ddot{q} \in \mathbb{R}^{2}$ are the vectors of joint angular position, velocity, and acceleration, respectively. $M(q)$ is the $2 \times 2$ symmetric positive-definite inertia matrix, $C(q, \dot{q})$ is the $2 \times 1$ vector containing Coriolis and centrifugal forces, $G(q)$ is the $2 \times 1$ gravitational torque, $\tau$ is $2 \times 1$ vector of applied joint torques that are actually the control inputs and $\tau_{d}$ denotes the $2 \times 1$ bounded input disturbance vector. Moreover, the involved matrices can be expressed as

$$
\begin{gathered}
M(q)=\left[\begin{array}{ll}
a_{11}(q) & a_{12}(q) \\
a_{21}(q) & a_{22}(q)
\end{array}\right], \\
C(q, \dot{q})=\left[\begin{array}{c}
-\beta_{12}\left(q_{2}\right) \dot{q}_{1}^{2}-2 \beta_{12}\left(q_{2}\right) \dot{q}_{1} \dot{q}_{2} \\
\beta_{12}\left(q_{2}\right) \dot{q}_{2}^{2}
\end{array}\right], \\
\tau_{d}=\left[\begin{array}{c}
\tau_{d 1} \\
\tau_{d 2}
\end{array}\right], \\
G(q)=\left[\begin{array}{c}
\left(m_{1}+m_{2}\right) g r_{1} \cos \left(q_{2}\right)+m_{2} g r_{2} \cos \left(q_{1}+q_{2}\right) \\
m_{2} g r_{2} \cos \left(q_{1}+q_{2}\right)
\end{array}\right], \\
\tau=\left[\begin{array}{c}
\tau_{1} \\
\tau_{2}
\end{array}\right], \\
a_{11}(q)=\left(m_{1}+m_{2}\right) r_{1}^{2}+m_{2} r_{2}^{2}+2 m_{2} r_{1} r_{2} \cos \left(q_{2}\right)+J_{1}, \\
a_{12}(q)=a_{21}(q)=m_{2} r_{2}^{2}+m_{2} r_{1} r_{2} \cos \left(q_{2}\right), \\
a_{22}(q)=m_{2} r_{2}^{2}+J_{2} .
\end{gathered}
$$

The system parameter values are $m_{1}=0.5 \mathrm{~kg}, m_{2}=1.5 \mathrm{~kg}$, $J_{1}=5 \mathrm{~kg} \mathrm{~m}, J_{2}=5 \mathrm{~kg} \mathrm{~m}, r_{1}=1 \mathrm{~m}$, and $r_{2}=0.8 \mathrm{~m}$. The parameters for FO-TSMC are defined as $p=3, q=$ $5, K_{1}=[5,10]^{T}, K_{2}=[5,10]^{T}, \lambda=[10,30]^{T}$, and $\alpha=$ 0.9. The parameters for FO-FTSMC are defined as $k_{1}=$ $15, k_{2}=6, a_{1}=2, a_{2}=3 / 5, \gamma_{1}=[5,10]^{T}$, and $\gamma_{2}=$ $[5,12]^{T}$. The parameters for FO-NFTSMC are defined as $k_{1}=$ $[5,10]^{T}, k_{2}=[10,30]^{T}, a_{1}=[2,2]^{T}, a_{2}=[5 / 3,5 / 3]^{T}$, 
$\gamma_{1}=[5,10]^{T}, \gamma_{2}=[10,35]^{T}, \rho=3 / 5, \tau_{d 1}=2 \sin (t)+$ $0.5 \sin (200 \pi t)$, and $\tau_{d 2}=\cos (2 t)+0.5 \sin (200 \pi t)$. The reference signal is set as $x_{d}=\left[1.25-7 e^{-t} / 5+7 e^{-4 t} / 20,1.25+\right.$ $\left.e^{-t}-e^{-4 t} / 4\right]^{T}$.

In order to compare the performance of the three controllers, the trajectories are listed in Figure 4(a), control efforts $\tau_{1}, \tau_{2}$ are shown in Figure 4(b), and the sliding surface $s_{1}, s_{2}$ are depicted in Figure 5. FO-NFTSMC and FO-FTSMC can keep the boundary layer within 0.1 while FO-TSMC is relatively large; the main possible cause of this phenomenon is that the sliding surface of FO-TSMC will need a larger control effort to guarantee the reaching law to track the desired trajectory. For the FO-NFTSMC and FO-FTSMC, the coupled term $\operatorname{sig}^{a_{i}} e \cdot \dot{e}(i=1,2)$ have the ability to attenuate the chattering whether $e \dot{e}$ is large or small. The simulation results show that the newly proposed method can eliminate the chattering by carefully tuning the parameters.

\section{Conclusion}

In this paper, the problem of designing FO-FTSMC and FO-NFTSMC for a class of uncertain dynamical systems is investigated. The uncertainties are considered in the system state matrices. Based on the Lyapunov stability criteria, the fractional-order terminal sliding mode control laws are designed and the finite time stability of the closed-loop systems is guaranteed under the proposed controller. By carefully chosing the tuning parameters, the simulation results have demonstrated the effectiveness and robustness of the proposed method. Besides, the two proposed controllers are compared with the FO-TSMC; results show that FO-FTSMC and FO-NFTSMC are superior to FO-TSMC.

\section{Acknowledgment}

This work was supported by the Education Department of Heilongjiang Province Science and Technology Research Projects.

\section{References}

[1] A. Oustaloup, "From fractality to non-integer derivation through recursivity, a property common to these two concepts: a fundamental idea from a new process control strategy," in Proceedings of 12th IMACS World Conference, pp. 203-208, Paris, France, 1988.

[2] I. Podlubny, Fractional Differential Equations, Academic Press, New York, NY, USA, 1999.

[3] I. Petráš, "Fractional calculus," in Fractional-Order Nonlinear Systems: Modeling, Analysis and Simulation, A. C. J. Luo and N. H. Ibragimov, Eds., pp. 7-42, Higher Education Press, Beijing, China, 2011.

[4] A. A. Kilbas, H. M. Srivastava, and J. J. Trujillo, Theory and Applications of Fractional Differential Equations, Elsevier B. V., Amsterdam, The Netherlands, 2006.

[5] I. Podlubny, "Fractional-order systems and $\mathrm{PI}_{\lambda} \mathrm{D}_{\mu}$-controllers," IEEE Transactions on Automatic Control, vol. 44, no. 1, pp. 208$214,1999$.
[6] C. A. Monje, A. J. Calderón, B. M. Vinagre, and V. Feliu, “The fractional order lead compensator," in Proceedings of the 2nd IEEE International Conference on Computational Cybernetics, vol. 1, pp. 347-352, Vienna, Austria, September 2004.

[7] O. P. Agrawal, "A general formulation and solution scheme for fractional optimal control problems," Nonlinear Dynamics, vol. 38, no. 1-4, pp. 323-337, 2004.

[8] C. Tricaud and Y. Chen, "An approximate method for numerically solving fractional order optimal control problems of general form," Computers and Mathematics with Applications, vol. 59, no. 5, pp. 1644-1655, 2010.

[9] Y. Li, Y. Chen, and I. Podlubny, "Mittag-Leffler stability of fractional order nonlinear dynamic systems," Automatica, vol. 45, no. 8, pp. 1965-1969, 2009.

[10] Y. Li, Y. Chen, and I. Podlubny, "Stability of fractional-order nonlinear dynamic systems: lyapunov direct method and generalized Mittag-Leffler stability," Computers and Mathematics with Applications, vol. 59, no. 5, pp. 1810-1821, 2010.

[11] S. Dadras and H. R. Momeni, "Control of a fractional-order economical system via sliding mode," Physica A, vol. 389, no. 12, pp. 2434-2442, 2010.

[12] M. P. Aghababa, "Robust stabilization and synchronization of a class of fractional-order chaotic systems via a novel fractional sliding mode controller," Communications in Nonlinear Science and Numerical Simulation, vol. 17, no. 6, pp. 2670-2681, 2012.

[13] E. A. Boroujeni and H. R. Momeni, "Non-fragile nonlinear fractional order observer design for a class of nonlinear fractional order systems," Signal Processing, vol. 92, no. 10, pp. 2365-2370, 2012.

[14] S. Saha, S. Das, S. Das, and A. Gupta, "A conformal mapping based fractional order approach for sub-optimal tuning of PID controllers with guaranteed dominant pole placement," Communications in Nonlinear Science and Numerical Simulation, vol. 17, no. 9, pp. 3628-3642, 2012.

[15] Y. Shen, S. Yang, H. Xing, and G. Gao, "Primary resonance of Duffing oscillator with fractional-order derivative," Communications in Nonlinear Science and Numerical Simulation, vol. 17, no. 7, pp. 3092-3100, 2012.

[16] J. Shen and J. Lama, "State feedback $H_{\infty}$ control of commensurate fractional-order systems," International Journal of Systems Science, vol. 45, no. 3, pp. 363-372, 2014.

[17] J. Shen and J. Lama, " $H_{\infty}$ model reduction for positive fractional order systems," Asian Journal of Control, 2013.

[18] D.-J. Wang and X.-L. Gao, " $\mathrm{H}_{\infty}$ design with fractional-order PD $\mu$ controllers," Automatica, vol. 48, no. 5, pp. 974-977, 2012.

[19] T. Binazadeh and M. H. Shafiei, "Output tracking of uncertain fractional-order nonlinear systems via a novel fractional-order sliding mode approach," Mechatronics, vol. 23, no. 7, Article ID 888892, 2013.

[20] J. Shen, J. Cao, and J. Lu, "Consensus of fractional-order systems with non-uniform input and communication delays," Proceedings of the Institution of Mechanical Engineers I, vol. 226, no. 2, pp. 271-283, 2012.

[21] J. Q. Lu, J. Shen, J. D. Cao, and J. Kurths, "Consensus and synchronization in complex networks," in Consensus of Networked Multi-agent Systems with Delays and Fractional-Order Dynamics, K. Ljupco, Ed., Understanding Complex Systems, pp. 69-110, Springer, Berlin, Germany, 2013.

[22] J. Shen and J. D. Cao, "Necessary and sufficient conditions for consensus of delayed fractional-order systems," Asian Journal of Control, vol. 14, no. 6, pp. 1690-1697, 2012. 
[23] Y. Tang, M. Cui, C. Hua, L. Li, and Y. Yang, "Optimum design of fractional order PI $\lambda \mathrm{D} \mu$ controller for AVR system using chaotic ant swarm," Expert Systems with Applications, vol. 39, no. 8, pp. 6887-6896, 2012.

[24] L. Yang and J. Yang, "Nonsingular fast terminal sliding-mode control for nonlinear dynamical systems," International Journal of Robust and Nonlinear Control, vol. 21, no. 16, pp. 1865-1879, 2011.

[25] M. Zhihong and X. H. Yu, "Terminal sliding mode control of MIMO linear systems," IEEE Transactions on Circuits and Systems I, vol. 44, no. 11, pp. 1065-1070, 1997.

[26] S. Yu, X. Yu, B. Shirinzadeh, and Z. Man, "Continuous finitetime control for robotic manipulators with terminal sliding mode," Automatica, vol. 41, no. 11, pp. 1957-1964, 2005.

[27] D. Z. Xu, B. Jiang, M. S. Qian, and J. Zhao, “Terminal sliding mode control using adaptive fuzzy-neural observer," Mathematical Problems in Engineering, vol. 2013, Article ID 958958, 8 pages, 2013.

[28] Y. Feng, X. Yu, and Z. Man, "Non-singular terminal sliding mode control of rigid manipulators," Automatica, vol. 38, no. 12, pp. 2159-2167, 2002.

[29] S. Dadras and H. R. Momeni, "Fractional terminal sliding mode control design for a class of dynamical systems with uncertainty," Communications in Nonlinear Science and Numerical Simulation, vol. 17, no. 1, pp. 367-377, 2012.

[30] D. Baleanu, "New treatise in fractional dynamics," in Complex Systems, L. C. J. Albert and J. Q. Sun, Eds., pp. 1-41, Higher Education Press, Beijing, China, 2011.

[31] H.-K. Chen, "Chaos and chaos synchronization of a symmetric gyro with linear-plus-cubic damping," Journal of Sound and Vibration, vol. 255, no. 4, pp. 719-740, 2003.

[32] G. Schweitzer, "Active magnetic bearings-chances and limitations," in Proceedings of the 6th IFToMM International Conference of Rotor Dynamics, vol. 1, pp. 1-4, Sydney, Australia, 2002.

[33] G. Schweitzer, H. Bleuler, and A. Traxler, Active Magnetic Bearings: Basics, Properties, and Application of Active Magnetic Bearings, VDF Hochschulverlag, Zurich, Switzerland, 1994.

[34] A. A. Zaher, "Nonlinear control of systems with multiple equilibria and unknown sinusoidal disturbance," Communications in Nonlinear Science and Numerical Simulation, vol. 12, no. 8, pp. 1518-1533, 2007. 


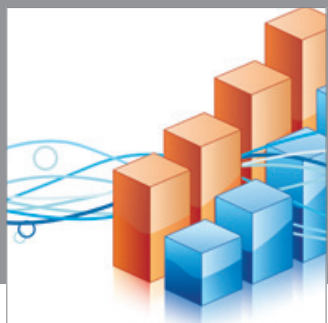

Advances in

Operations Research

mansans

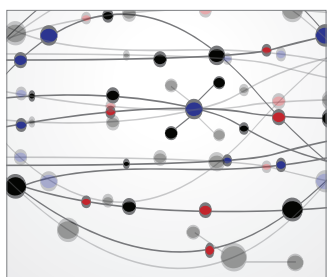

The Scientific World Journal
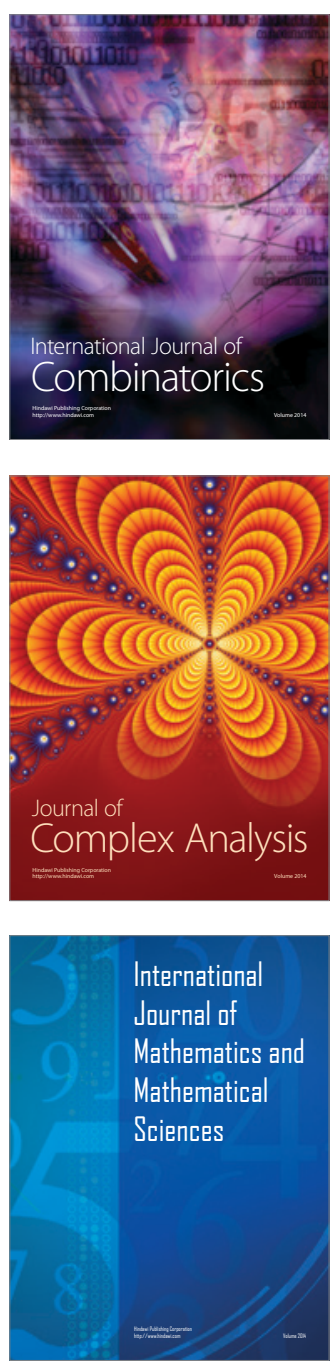
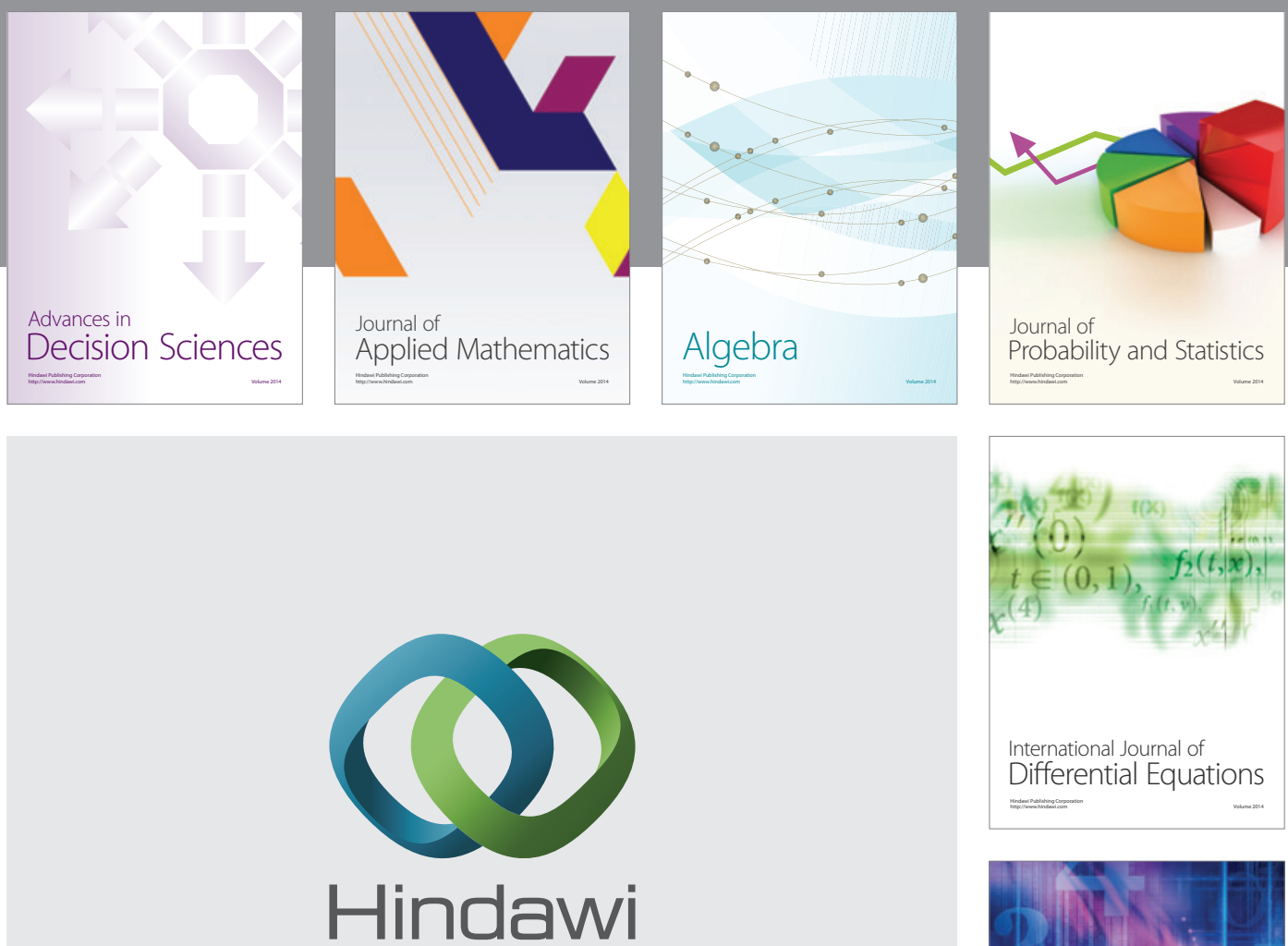

Submit your manuscripts at http://www.hindawi.com
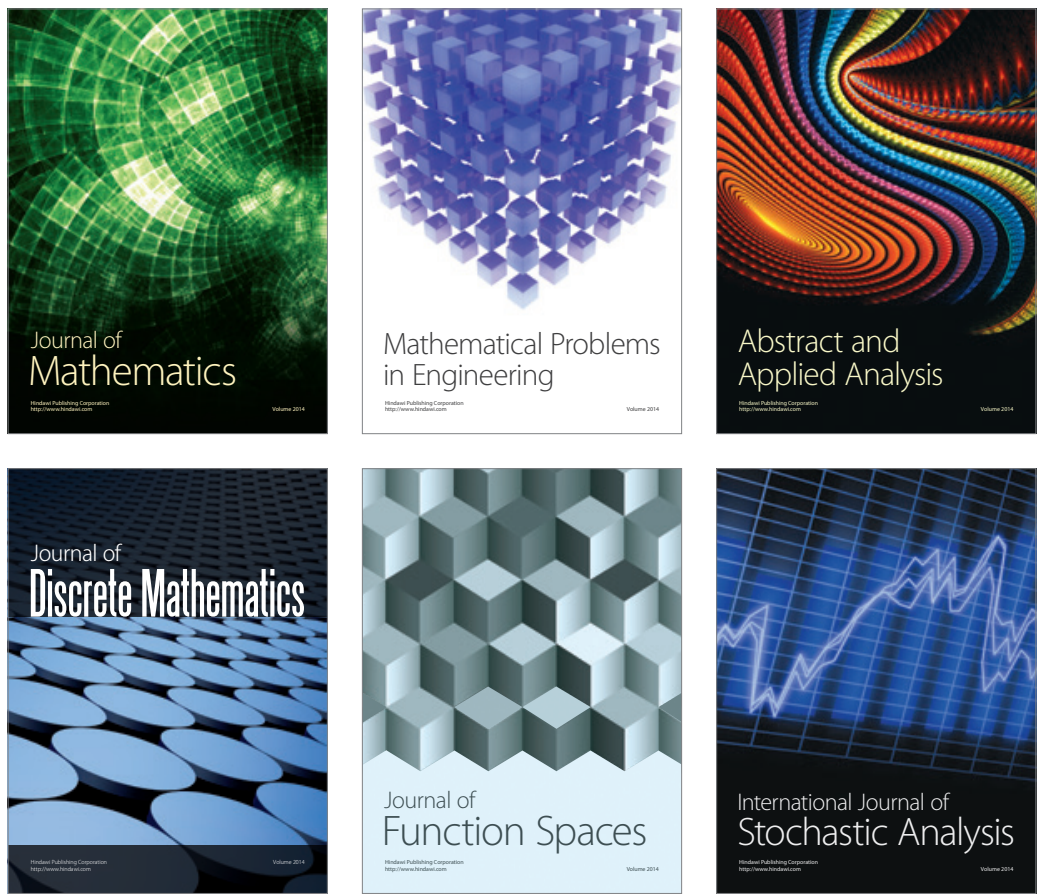

Journal of

Function Spaces

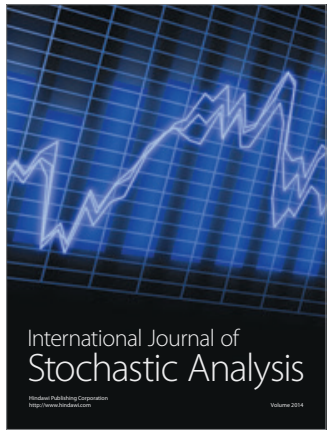

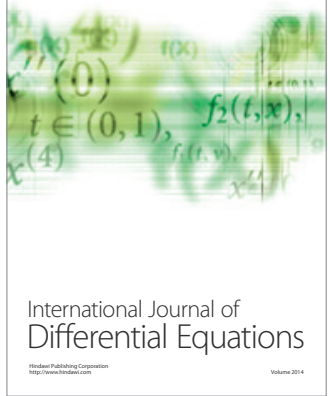
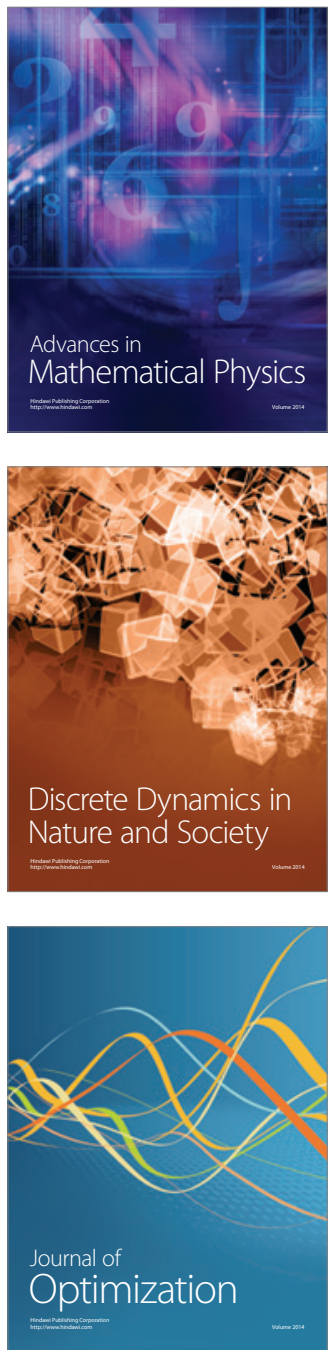